\title{
Modelación de la tasa de respiración del mango cortado osmodeshidratado en un sistema cerrado
}

\author{
José E. Zapata ${ }^{*}$, Camilo Agudelo-Cuartas ${ }^{1}$, y Claudia E. Restrepo ${ }^{2}$ \\ (1) Facultad de Ciencias Farmacéuticas y Alimentarias. Grupo de Nutrición y Tecnología de Alimentos. Universidad de \\ Antioquia, Medellín, Colombia. (correo-e: edgar.zapata@udea.edu.co). \\ (2) Facultad de Ciencias de la Salud. Grupos de investigación en SFC. Corporación Universitaria Uniremington. Medellín, \\ Colombia (correo- e: claudia.restrepo@uniremington.edu.co).
}

* Autor a quien debe ser dirigida la correspondencia

Recibido Jun. 18, 2020; Aceptado Ago. 18, 2020; Versión final Sep. 14, 2020, Publicado Feb. 2021

\section{Resumen}

El objetivo de esta investigación es modelar la velocidad de respiración (VR) del mango (Mangifera indica L.), variedad Tommy Atkins, cortado osmodeshidratado a tres temperaturas en un sistema cerrado. Mangos cortados en cubos se osmodeshidrataron en solución de sacarosa $\left(60^{\circ}\right.$ Brix) a $323.15 \mathrm{~K}$ durante 4 horas. La concentración de $\mathrm{O}_{2}$ y $\mathrm{CO}_{2}$ fue medida en las condiciones establecidas. La VR experimental fue calculada y ajustada a los modelos de regresión (M-R), Michaelis-Menten (M-M), Arrhenius y a un modelo global semiempírico que predice la concentración de gases en función del tiempo y temperatura. Los resultados muestran que la VR predicha con M-M presentó mayores ajustes comparado con M-R. La energía de activación fue 55.76 y $31.42 \mathrm{kJg}^{-1} \mathrm{~h}^{-1}$ para $\mathrm{O}_{2}$ y CO 2 , respectivamente. El modelo global predijo la concentración del $\mathrm{O}_{2}\left(\mathrm{R}^{2}=\right.$ $0.96)$ y del $\mathrm{CO}_{2}\left(\mathrm{R}^{2}=0.96\right)$. Se concluye que la osmodeshidratación disminuyó la VR experimental comparada con otros estudios en mango entero y cortado fresco.

Palabras clave: Mangifera indica L.; velocidad de respiración; osmodeshidratación; Michaelis-Menten; ecuación de Arrhenius

\section{Respiration rate modeling of osmodehydrated cut mango in a closed system}

\begin{abstract}
The primary objective of this research study is to model the respiration rate (RR) of osmodehydrated cut mango (Mangifera indica L.), Tommy Atkins variety, at three temperatures $(277.15,293.15$ and $308.15 \mathrm{~K}$ ) in a closed system. Mangos cut in cubes were osmodehydrated in a sucrose solution $\left(60^{\circ}\right.$ Brix) at $323.15 \mathrm{~K}$ for 4 hours. $\mathrm{O}_{2}$ and $\mathrm{CO}_{2}$ concentrations were measured at established conditions. The experimental RR was calculated and fitted to the regression model (R-M), the Michaelis-Menten (M-M) and Arrhenius models, and a global semiempirical model that predicts gas concentrations as a function of time and temperature. The results show that M-M's predicted RR showed greater fitting than that of R-M. The activation energy was 55.76 and $31.42 \mathrm{kJg}$ 'h ${ }^{-1}$ for $\mathrm{O}_{2}$ and $\mathrm{CO}_{2}$, respectively. The global model predicted $\mathrm{O}_{2}\left(\mathrm{R}^{2}=0.96\right)$ and $\mathrm{CO}_{2}\left(\mathrm{R}^{2}=0.96\right)$ concentrations. It is concluded that osmotic dehydration decreased experimental RR when compared to that of previous studies examining both whole and fresh cut mango.
\end{abstract}

Keywords: Mangifera indica L.; respiration rate; osmotic dehydration; Michaelis-Menten; Arrhenius equation 


\section{INTRODUCCIÓN}

Es indiscutible la importancia que tiene la respiración en los procesos de conservación de frutas y vegetales cosechados, debido a que se relaciona con diferentes reacciones de degradación de la pared celular, la reducción de su contenido de ácidos orgánicos, la síntesis del flavor, la fermentación y la peroxidación de lípidos de la membrana por formación de especies reactivas de oxígeno, resultando en estrés oxidativo (Ntsoane et al. 2019). A pesar de que este proceso varía según la especie, variedad, temperatura de almacenamiento y estado fisiológico del vegetal (Nicolaï et al., 2009), en general, una alta velocidad de respiración implica un metabolismo acelerado y está asociada con una vida corta en almacenamiento, por lo que controlar la velocidad de respiración permite regular el metabolismo y de esa forma extenderse la vida en almacenamiento del vegetal (Ghosh y Dash, 2018).

Cambios en la composición de la atmósfera en la que se encuentra un alimento, pueden reducir su velocidad de respiración y prolongar su vida útil, inhibiendo o retrasando las reacciones de deterioro (Selcuk y Erkan, 2015). Es así como se ha reportado que la disminución en la concentración de $\mathrm{O}_{2}$ retarda el metabolismo de productos como papaya (Carvajal et al., 2004), brócoli, zanahorias, peras, tomates (Nicolaï et al., 2009) y mango en diferentes presentaciones (Agudelo et al., 2016), y que el $\mathrm{CO}_{2}$ puede actuar como supresor de la respiración en banana (Bhande et al., 2008), manzanas y brócoli (Nicolaï et al., 2009) y mango (Agudelo et al., 2016).

Los efectos negativos del oxígeno se deben principalmente a que la acumulación de oxígeno reactivo daña la integridad de la membrana mitocondrial, resultando finalmente en una disfunción mitocondrial irreversible, lo cual se cree que es la principal causa de la senescencia en diferentes tipos de organismos, entre los que se encuentran las frutas postcosecha (Qin et al., 2009). Por otro lado, la temperatura tiene efecto sobre todas las reacciones bioquímicas durante la madurez del vegetal, por lo que afecta de forma directa su calidad durante el almacenamiento y también puede favorecer el crecimiento de ciertos microorganismos (Nicolaï et al., 2009). Además de ciertos efectos sobre algunas piezas de mango cortado debido a la generación de color oscuro en los vasos alrededor de los cortes, por acción de la enzima polifenol oxidasa sobre los compuestos fenólicos liberados (Blanco-Díaz et al., 2016).

En general el corte afecta las características fisicoquímicas y fisiológicas de la fruta, por el efecto de la mayor acción enzimática debida a la liberación de los jugos celulares y la salida de agua libre, con el incremento de la actividad acuosa del producto, al tener el agua más disponible para el desarrollo de microorganismos y la acción enzimática, con lo cual se acorta su vida útil (Pan et al., 2015). Por otro lado, el corte incrementa los niveles de transpiración y la pérdida de compuestos aromáticos típicos del producto (Buitrago-Dueñas et al., 2019), así como alteraciones de la textura y del color, que han sido asociados al deterioro fisiológico, que probablemente surge de la plasmólisis de las células vegetales (Pan et al., 2015).

Una de las alternativas que se ha utilizado para prolongar la vida útil de productos cortados ha sido la deshidratación osmótica (DO), la cual reduce parcialmente el contenido de agua en la fruta, disminuyendo la actividad acuosa y la posibilidad de deterioro fisicoquímico y microbiológico (Arias et al., 2017). El tratamiento osmótico consiste en la inmersión de la fruta en una solución hipertónica donde la pared celular actúa como una membrana selectiva semipermeable, que favorece la remoción parcial de agua y la retención del color, aroma y sabor de la fruta (Sanjinez-Argandoña et al., 2018). Sin embargo, este tratamiento tiene efectos sobre la respiración del vegetal, porque puede generar la formación una barrera de soluto en la superficie del producto (Arias et al., 2017), con lo que la transferencia de gases se modifica, lo que hace necesario estudiar las condiciones de respiración posteriores al proceso de deshidratación.

La obtención de modelos del proceso respiratorio es un importante paso en el diseño y selección de sistemas de empaque y almacenamiento de productos hortofrutícolas como es el caso del empacado en atmósferas modificadas (Agudelo et al., 2016). El efecto de la temperatura se ha evaluado mediante la ecuación de Arrhenius siendo este un aspecto de primera importancia en la conservación de productos alimenticios (Ghosh y Dash, 2018). Por otro lado, el modelo de Michaelis-Menten (M-M) usa principios de inhibición no competitiva de la cinética enzimática, donde se supone que el $\mathrm{CO}_{2}$ reacciona con un complejo enzima-sustrato (Bhande et al., 2008), el cual ha tenido resultados predictivos en diversos productos como mango entero (Zapata et al., 2018), bananito (Castellanos, 2012), pimiento rojo (Artés-Hernández et al., 2010), banano (Bhande et al., 2008), mango (Ravindra y Goswami, 2008), entre otras frutas.

En nuestro medio son escasos los trabajos orientados a evaluar este tipo de parámetros en frutas y vegetales. Reconociendo que el modelamiento de la tasa de respiración con todos los factores implicados en la reacción enzimática sería de muy alta complejidad y poco práctico, la estrategia usual ha sido desarrollar modelos 
empíricos para cada producto como una función de variables controlables, como la temperatura o la concentración gaseosa (Agudelo et al., 2016; Bhande et al., 2008). En base a esto, el objetivo del presente trabajo fue evaluar diferentes modelos matemáticos en la predicción de la tasa de respiración de mango cortado osmodeshidratado var. Tommy Atkins, a diferentes temperaturas en un sistema cerrado.

\section{MATERIALES Y MÉTODOS}

La metodología se presenta en varias subsecciones, para una mayor claridad: preparación de la materia prima, proceso de deshidratación osmótica y modelamiento de la tasa de respiración.

\section{Preparación de la materia prima}

Se trabajó con mango (Mangifera indica L.), adquirido en la ciudad de Medellín-Colombia. Se utilizó la variedad Tommy Atkins en estado de madurez en escala de color entre 1 y 2, con valores de peso, sólidos solubles y acidez titulable, entre 350 - $550 \mathrm{~g}, 7.6$ - $8.5^{\circ}$ Brix y 0.97 - $0.83 \%$ de ácido cítrico, respectivamente, de acuerdo a la norma Norma Técnica Colombiana 5210 (ICONTEC, 2003). Se lavaron con detergente alcalino y se desinfectaron con solución de hipoclorito de sodio (100 ppm). Finalmente, se cortaron en cubos de $10-15 \mathrm{~mm}$ de arista, descartando la semilla y epidermis.

\section{Proceso de deshidratación osmótica (DO) del mango}

Los cubos de mango fresco se sumergieron en solución de sacarosa a $60^{\circ}$ Brix en relación 1:5 (fruta:solución), la cual se mantuvo a $323.15 \mathrm{~K}$ durante 4 horas. Se extrajeron muestras en intervalos de 1 hora, se enjuagaron con agua destilada y se secaron con papel absorbente para determinar los porcentajes de pérdida de agua (\% PA) (Arias et al., 2017) y ganancia de sólidos solubles (\%GS) de acuerdo a las ecuaciones (1) y (2), previamente utilizadas por Agudelo et al. (2016).

$$
\begin{aligned}
& \% P A=\frac{\left(M_{i} x_{w i}-M_{f} x_{w f}\right)}{M_{i}} \times 100 \\
& \% G S=\frac{\left(M_{i} x_{w i}-M_{f} x_{w f}\right)}{M_{i}} \times 100
\end{aligned}
$$

Una vez finalizado el proceso de DO se determinó por triplicado, el porcentaje de humedad utilizando una estufa de aire forzado (Thermo Scientific, USA) según el método oficial AOAC 934.06 (AOAC, 1984), el porcentaje de sólidos solubles expresado como ${ }^{\circ}$ Brix con un refractómetro digital Atago (Milton Roy Company, USA), acidez titulable expresada como \% de ácido cítrico por el método AOAC 942.15 (AOAC, 1984) y el pH se midió con ayuda de un pHmetro Laqua F-71G ( \pm 0.01$)$ (Horiba Scientific, Japón).

\section{Cálculo de velocidad de respiración experimental}

El montaje del experimento para el cálculo de la velocidad de respiración se realizó conforme a Ravindra y Goswami, (2008) con algunas modificaciones; se depositaron $0.15 \mathrm{~kg}$ de mango osmodeshidratado en un recipiente hermético de vidrio con capacidad de $600 \mathrm{~mL}$, el cual se encontraba a condiciones atmosféricas iniciales de $20.94 \%$ de $\mathrm{O} 2,78.08 \%$ de N2 y $0.03 \%$ de CO2, el experimento se realizó a $277.15 \mathrm{~K}, 293.15 \mathrm{~K}$ y $308.15 \mathrm{~K}$ respectivamente, cada una por triplicado. Las concentraciones de $\mathrm{O} 2$ y $\mathrm{CO} 2$ se midieron en función del tiempo a través de un septum de goma en la tapa, utilizando un Analizador de gases PBI Check Point O2/CO2 (Dansensor, Dinamarca). Para el cálculo de la velocidad de respiración se utilizaron las ecuaciones (3) y (4), las cuales ya han sido utilizadas en productos como pimiento rojo (Artés Hernández et al., 2010), mango (Zapata et al., 2018), banana (Bhande et al., 2008).

$$
\begin{aligned}
& \mathrm{R}_{\mathrm{O}_{2}}=\left\{\frac{\left[\mathrm{O}_{2}\right]_{\mathrm{t}}-\left[\mathrm{O}_{2}\right]_{\mathrm{t}+1}}{\Delta \mathrm{t}}\right\} \frac{\mathrm{V}}{\mathrm{W}} \\
& \mathrm{R}_{\mathrm{CO}_{2}}=\left\{\frac{\left[\mathrm{CO}_{2}\right]_{\mathrm{t}+1}-\left[\mathrm{CO}_{2}\right]_{\mathrm{t}}}{\Delta \mathrm{t}}\right\} \frac{\mathrm{V}}{\mathrm{W}}
\end{aligned}
$$




\section{Modelamiento del comportamiento de los gases}

El comportamiento de los gases se modeló empleando las ecuaciones (5) y (6) ajustando las concentraciones de $\mathrm{O}_{2}$ y $\mathrm{CO}_{2}$ respectivamente, en función del tiempo. El ajuste de los datos experimentales a las ecuaciones 5 y 6 se realizó por regresión no lineal con el software Matlab R2011a.

$$
\begin{aligned}
& {\left[\mathrm{CO}_{2}\right]=\frac{\mathrm{t}}{(\mathrm{at}+\mathrm{b})}} \\
& {\left[\mathrm{O}_{2}\right]=0,21-\frac{\mathrm{t}}{(\mathrm{at}+\mathrm{b})}}
\end{aligned}
$$

\section{Modelamiento de la velocidad de respiración}

Se realizó utilizando los modelos de regresión (M-R) y de Michaelis-Menten (M-M), descritos a continuación:

\section{Modelo de Regresión (M-R)}

En este modelo se derivan las ecuaciones (5) y (6) para obtener las ecuaciones (7) y (8) (Ravindra y Goswami, 2008).

$$
\begin{aligned}
& \frac{\mathrm{d}\left[\mathrm{CO}_{2}\right]}{\mathrm{dt}}=\frac{\text { at }}{(\mathrm{at}+\mathrm{b})^{2}}+\frac{1}{(\mathrm{at}+\mathrm{b})} \\
& \frac{\mathrm{d}\left[\mathrm{O}_{2}\right]}{\mathrm{dt}}=\frac{\mathrm{at}}{(\mathrm{at}+\mathrm{b})^{2}}-\frac{1}{(\mathrm{at}+\mathrm{b})}
\end{aligned}
$$

Las ecuaciones (7) y (8) se reemplazan en las ecuaciones (3) y (4), para calcular la tasa de respiración, como sigue:

$$
\begin{aligned}
& \mathrm{R}_{\mathrm{O}_{2}}=\left\{\frac{\mathrm{at}}{(\mathrm{at}+\mathrm{b})^{2}}-\frac{1}{(\mathrm{at}+\mathrm{b})}\right\} \frac{\mathrm{V}}{\mathrm{W}} \\
& \mathrm{R}_{\mathrm{CO}_{2}}=\left\{\frac{1}{(a \mathrm{at}+b)}-\frac{\mathrm{at}}{(a t+b)^{2}}\right\} \frac{\mathrm{V}}{\mathrm{W}}
\end{aligned}
$$

\section{Modelo de Michaelis-Menten (M-M)}

El modelo de M-M se representa a través de las ecuaciones (11) y (12), las cuales expresan un mecanismo de inhibición no competitiva de la cinética enzimática en términos de la velocidad de consumo de $\mathrm{O}_{2}$ y velocidad de producción de $\mathrm{CO}_{2}$ (Ghosh y Dash, 2018).

$$
\begin{aligned}
& \mathrm{R}_{\mathrm{O}_{2}}=\frac{\mathrm{V}_{\mathrm{mo}}\left[\mathrm{O}_{2}\right]}{\mathrm{K}_{\mathrm{mo}}+\left[1+\frac{\left[\mathrm{CO}_{2}\right]}{\mathrm{K}_{\mathrm{io}}}\right]\left[\mathrm{O}_{2}\right]} \\
& \mathrm{R}_{\mathrm{CO}_{2}}=\frac{\mathrm{V}_{\mathrm{mc}}\left[\mathrm{O}_{2}\right]}{\mathrm{K}_{\mathrm{mc}}+\left[1+\frac{\left[\mathrm{CO}_{2}\right]}{\mathrm{K}_{\mathrm{ic}}}\right]\left[\mathrm{O}_{2}\right]}
\end{aligned}
$$

Las tasas de respiración se estimaron mediante la linealización de las ecuaciones (11) y (12) obteniendo las ecuaciones (13) y (14), a las que se aplicó una regresión lineal múltiple con el software estadístico R, versión 2.15.0 (The R Foundation for Statistical Computing, Austria). 


$$
\begin{aligned}
& \frac{1}{\mathrm{R}_{\mathrm{O}_{2}}}=\frac{1}{\mathrm{~V}_{\mathrm{mo}}}+\frac{\mathrm{k}_{\mathrm{mo}}}{\mathrm{V}_{\mathrm{mo}}} \frac{1}{\left[\mathrm{O}_{2}\right]}+\frac{1}{\mathrm{k}_{\mathrm{io}} \mathrm{V}_{\mathrm{mo}}}\left[\mathrm{CO}_{2}\right] \\
& \frac{1}{\mathrm{R}_{\mathrm{CO}_{2}}}=\frac{1}{\mathrm{~V}_{\mathrm{mc}}}+\frac{\mathrm{k}_{\mathrm{mc}}}{\mathrm{V}_{\mathrm{mc}}} \frac{1}{\left[\mathrm{O}_{2}\right]}+\frac{1}{\mathrm{k}_{\mathrm{ic}} \mathrm{V}_{\mathrm{mc}}}\left[\mathrm{CO}_{2}\right]
\end{aligned}
$$

\section{Efecto de la temperatura sobre la velocidad de respiración}

Para estimar el efecto de la temperatura en las velocidades de consumo de $\mathrm{O}_{2}$ y producción de $\mathrm{CO}_{2}$, se utilizó la ecuación de Arrhenius (15) previamente utilizada por Ghosh y Dash, en 2018.

$$
\mathrm{R}_{\mathrm{gas}}=\mathrm{R}_{\mathrm{p}} \exp \left[-\frac{\mathrm{E}_{\mathrm{a}}}{\mathrm{RT} \mathrm{T}_{\mathrm{abs}}}\right]
$$

Estimación de la concentración de los gases en función del tiempo y la temperatura

Una vez conocido los resultados del modelo de Arrhenius se evalúo un modelo global con el que se estimó la concentración de los gases $\mathrm{O}_{2}$ y $\mathrm{CO}_{2}$ en función de la temperatura y el tiempo representado por las ecuaciones (16) y (17) (Iqbal et al., 2009). Este tipo de ecuación minimiza los errores en el cálculo de los parámetros.

$$
\begin{aligned}
& {\left[\mathrm{O}_{2}\right]=\left[\mathrm{O}_{2}\right]_{\mathrm{O}_{2}}^{\mathrm{O}_{2}}-\mathrm{R}_{\mathrm{p}_{\mathrm{O} 2}} \exp \left[-\frac{\mathrm{E}_{\mathrm{aO} 2}}{\mathrm{R}} \times\left(\frac{1}{\mathrm{~T}}-\frac{1}{\mathrm{~T}_{\text {ref }}}\right)\right] \times \frac{\mathrm{W}}{\mathrm{V}_{\mathrm{f}}} \times\left(\mathrm{t}-\mathrm{t}_{\mathrm{i}}\right) \times 100} \\
& {\left[\mathrm{CO}_{2}\right]=\left[\mathrm{CO}_{2}\right]_{\mathrm{CO}_{2}}^{\mathrm{i}_{2}}+\mathrm{R}_{\mathrm{P}_{\mathrm{CO} 2}} \exp \left[-\frac{\mathrm{E}_{\mathrm{aCO} 2}}{\mathrm{R}} \times\left(\frac{1}{\mathrm{~T}}-\frac{1}{\mathrm{~T}_{\text {ref }}}\right)\right] \times \frac{\mathrm{W}}{\mathrm{V}_{\mathrm{f}}} \times\left(\mathrm{t}-\mathrm{t}_{\mathrm{i}}\right) \times 100}
\end{aligned}
$$

La energía de activación y el factor pre exponencial de las ecuaciones 15, 16 y 17 para cada gas se determinó a través del algoritmo de Levenberg-Marquardt utilizando el software Matlab ® (Mathworks Inc, MA, EUA) (Agudelo et al., 2016).

\section{RESULTADOS}

La discusión de los resultados sigue el mismo orden de las subsecciones planteadas en la metodología: deshidratación osmótica del mango cortado, respiración del mango en sistema cerrado, comportamiento de las velocidades de respiración, velocidades de respiración estimadas, y efecto de la temperatura sobre la velocidad de respiración.

\section{Deshidratación Osmótica (DO) del mango cortado}

Los parámetros cinéticos presentados en la Tabla 1 demuestran que la DO provocó una disminución de humedad del mango ocasionada por la difusión de agua desde la fruta hacia el jarabe de sacarosa y entrada de solutos del jarabe a la fruta, y una posible pérdida mínima de solutos propios del mango y otras moléculas sencillas como ciertos ácidos o aromas (Arias et al., 2017). Esa transferencia de masas también causó una disminución del tamaño y del peso de los cubos de mango, una pérdida de agua por encima del $60 \% \mathrm{~m} / \mathrm{m}$ y una ganancia de sólidos por encima del $4 \% \mathrm{~m} / \mathrm{m}$, en las $4 \mathrm{~h}$ de tratamiento.

Tabla 1: Parámetros cinéticos de la osmodeshidratación de cubos de mango

\begin{tabular}{|c|c|c|}
\hline Tiempo $(\mathrm{h})$ & $P A(\% \mathrm{~m} / \mathrm{m})$ & $G S(\% \mathrm{~m} / \mathrm{m})$ \\
\hline 1 & 49.86 & 0.82 \\
\hline 2 & 55.86 & 3.97 \\
\hline 3 & 64.09 & 3.62 \\
\hline 4 & 61.44 & 4.64 \\
\hline
\end{tabular}


Los resultados del porcentaje de humedad, sólidos solubles, acidez titulable y $\mathrm{pH}$ del mango OD correspondieron a $52.20 \pm 1.30 \%, 26,00 \pm 1.0^{\circ}$ Brix, $0.17 \pm 0.08 \%$ ácido cítrico y $5.09 \pm 0.10$, respectivamente. Los resultados de sólidos solubles coinciden con los reportados por Zuluaga et al. (2010) para mango OD var. Tommy Atkins (23 - 29 ํㅏix).

\section{Respiración del mango OD en sistema cerrado}

El consumo de $\mathrm{O}_{2}$ y producción de $\mathrm{CO}_{2}$ del mango $\mathrm{OD}$ se representa en la Figura 1, y en ella se observa como la temperatura afecta el comportamiento de los gases, siendo mayor la variación para la temperatura más alta $(308.15 \mathrm{~K})$. El cambio en las concentraciones de los gases $\mathrm{O}_{2}$ y $\mathrm{CO}_{2}$ para los cubos de mango $\mathrm{OD}$ siguió la misma tendencia (Figura 1), pero en menor magnitud que los reportados para mango Tommy Atkins entero (Zapata et al., 2018) y cortado fresco (Agudelo et al., 2016) en sistema cerrado; posiblemente por la capa de sacarosa que tiende a formarse en la superficie del producto con el ingreso de sólidos durante la OD (Arias et al., 2017), la cual tiende a ralentizar el proceso de respiración de las frutas cortadas según lo demostró Chiumarelli et al. (2011).
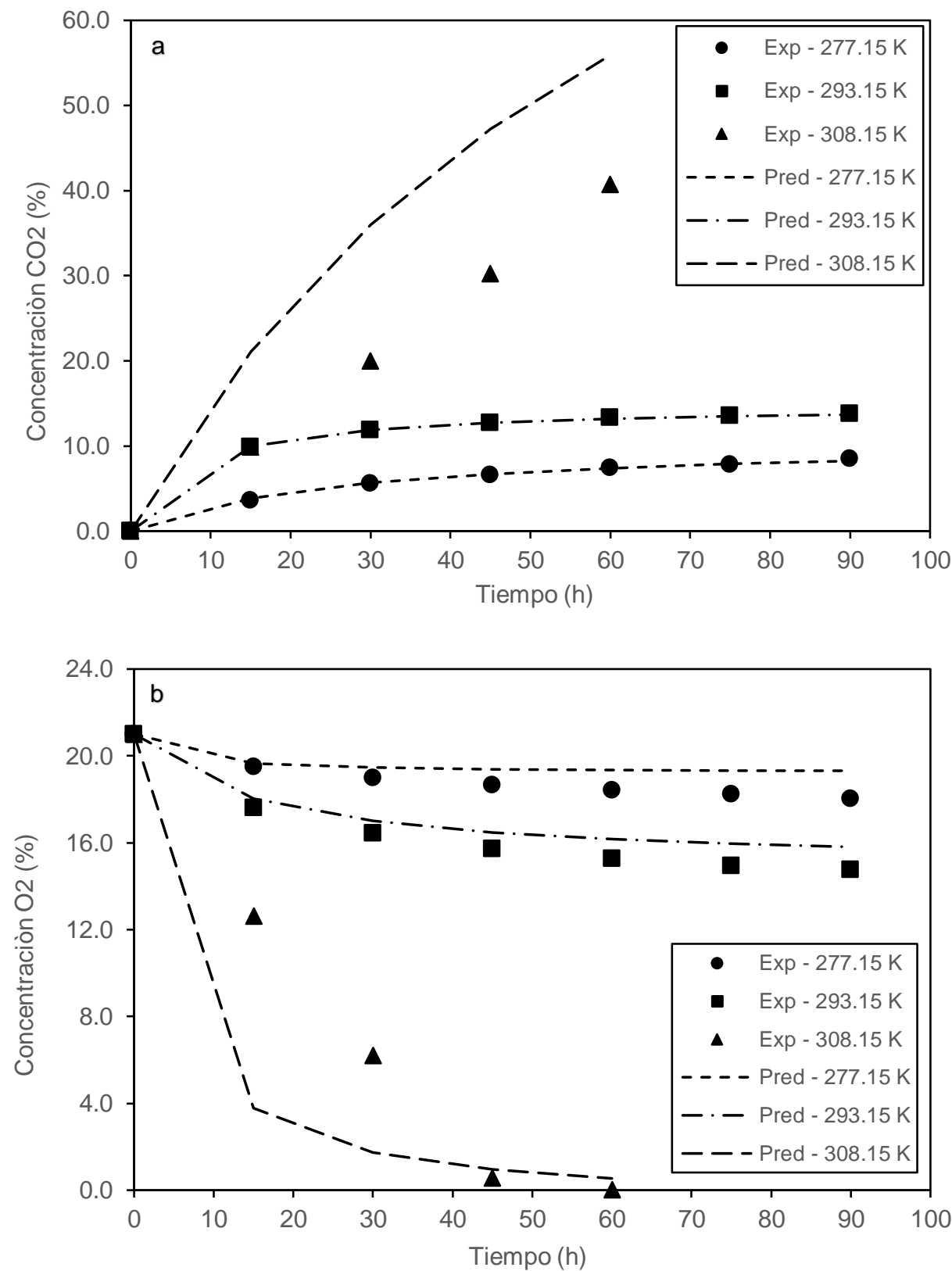

Fig. 1: Consumo de O2 (a) y producción de CO2 (b) del mango OD a diferentes temperaturas: • 277.15 K, • $293.15 \mathrm{~K}$ y $\Delta$ 308.15 K. Los marcadores representan los datos experimentales y las líneas punteadas la aproximación del modelo. 
Los coeficientes a y b de las ecuaciones (5) y (6) calculados para predecir el comportamiento de la concentración de $\mathrm{O}_{2}$ y $\mathrm{CO}_{2}$ en función del tiempo, se vieron afectados por la temperatura en ambos gases, de forma que, el incremento de temperatura redujo los valores de cada coeficiente, siendo más afectado el coeficiente b (Tabla 2). Este comportamiento también ha sido observado con banana (Ghosh y Dash, 2018), guayaba (Mangaraj y Goswami, 2011) y mango (Ravindra y Goswami, 2008). En consecuencia, los valores de coeficiente de determinación $\left(R^{2}\right)$ presentaron ajustes mayores a 0.65 para ambos gases y se procedió a utilizar dichos coeficientes para predecir los valores de las velocidades de respiración Ro2 y Rco2 representadas por las ecuaciones (9) y (10).

\section{Comportamiento de las velocidades de respiración}

Una comparación entre los valores iniciales del consumo de $\mathrm{O}_{2}\left(\mathrm{R}_{\circ}\right)$ y la producción de $\mathrm{CO}_{2}\left(\mathrm{R}_{\mathrm{co}}\right)$ para mango Tommy Atkins cortado OD, entero (Zapata et al., 2018) y cortado fresco (Agudelo et al., 2016) a las mismas temperaturas se presentan en la tabla 3.

Tabla 2: Coeficientes del modelo de regresión para mango osmodeshidratado, ajustados con las ecuaciones (5) y (6)

\begin{tabular}{|c|c|c|c|c|}
\hline Temperatura (K) & Gas & \multicolumn{4}{|c|}{ Modelo de regresión $(M-R)$} \\
\cline { 3 - 5 } & & $a$ & $b$ & $R^{2}$ \\
\hline \multirow{2}{*}{277.15} & $\mathrm{CO}_{2}$ & 9.37 & 249.60 & 0.67 \\
\cline { 2 - 5 } & $\mathrm{O}_{2}$ & 56.53 & 254.00 & 0.71 \\
\hline \multirow{2}{*}{293.15} & $\mathrm{CO}_{2}$ & 6.77 & 48.82 & 0.70 \\
\cline { 2 - 5 } & $\mathrm{O}_{2}$ & 16.40 & 257.10 & 0.76 \\
\hline \multirow{2}{*}{308.15} & $\mathrm{CO}_{2}$ & 0.80 & 59.29 & 0.93 \\
\cline { 2 - 5 } & $\mathrm{O}_{2}$ & 4.58 & 18.45 & 0.66 \\
\hline
\end{tabular}

Tabla 3: Comparación entre las velocidades iniciales de respiración experimentales para mango osmodeshidratado, mango entero y mango cortado.

\begin{tabular}{|c|c|c|c|c|c|c|}
\hline $\begin{array}{c}\text { Temperatura } \\
(K)\end{array}$ & \multicolumn{2}{|c|}{$\begin{array}{c}\text { Mango } \\
\text { Osmodeshidratado } \\
\left(\mathrm{mL} \mathrm{kg}^{-1} \mathrm{~h}^{-1}\right)\end{array}$} & \multicolumn{2}{|c|}{$\begin{array}{c}\text { Mango Entero } \\
(\text { Zapata et al., 2018) } \\
\left(\mathrm{mL} \mathrm{kg}^{-1} \mathrm{~h}^{-1}\right)\end{array}$} & \multicolumn{2}{|c|}{$\begin{array}{c}\text { Mango Cortado } \\
\text { Agudelo et al., 2016 } \\
\left(\mathrm{mL} \mathrm{kg}^{-1} \mathrm{~h}^{-1}\right)\end{array}$} \\
\hline & $\mathrm{R}_{\mathrm{O} 2}$ & $\mathrm{R}_{\mathrm{CO} 2}$ & $\mathrm{R}_{\mathrm{O} 2}$ & $\mathrm{R}_{\mathrm{CO} 2}$ & $\mathrm{R}_{\mathrm{O} 2}$ & $\mathrm{R}_{\mathrm{CO} 2}$ \\
\hline 277.15 & 1.30 & 7.00 & 12.52 & 13.00 & 5.43 & 5.11 \\
\hline 293.15 & 6.21 & 18.00 & 27.43 & 26.30 & 49.99 & 59.98 \\
\hline 308.15 & 15.37 & 32.00 & 44.31 & 58.10 & 90.01 & 115.10 \\
\hline
\end{tabular}

La diferencia entre estas velocidades puede atribuirse a que el mango entero mantiene la integridad de sus organelos y reduce las alteraciones fisiológicas a las que se somete el producto al momento del corte (Pan et al., 2015), por esta razón mantiene un ritmo respiratorio menor que el mango cortado (Zapata et al., 2018). No obstante, presenta una mayor velocidad de respiración que el mango OD. Por otro lado, en el mango cortado fresco no se produce la capa de sacarosa generada por el intercambio osmótico durante la OD, por tanto no se reduce la difusión de los gases en el material vegetal (Arias et al., 2017). Ríos et al. (2005) han observado el mismo comportamiento de formación de una capa de sacarosa y reducción en la difusión de los gases en papaya, por el proceso de OD. De acuerdo con estos autores, la diferencia en Ro2 y Rco2 en función de la temperatura, se debe al efecto que esta variable tiene sobre el metabolismo de los vegetales en general.

Las figuras 2, 3 y 4, representan el comportamiento de las velocidades de respiración para el mango cortado OD a diferentes temperaturas, así como el ajuste de los diferentes modelos propuestos. Se observa que, entre el tiempo inicial y final de medición para las diferentes temperaturas evaluadas, Roz disminuyó entre un 30 a $60 \%$, mientras que Rco2 redujo entre un 15 a $57 \%$. Esto se explica por el incremento de la concentración de $\mathrm{CO}_{2}$ y reducción de $\mathrm{O}_{2}$, siendo el primero un producto de la reacción y el segundo un reactante y bajo el principio de Le Chatelier, la velocidad de la reacción se reduce por ambas vías (Devanesan et al., 2011). 


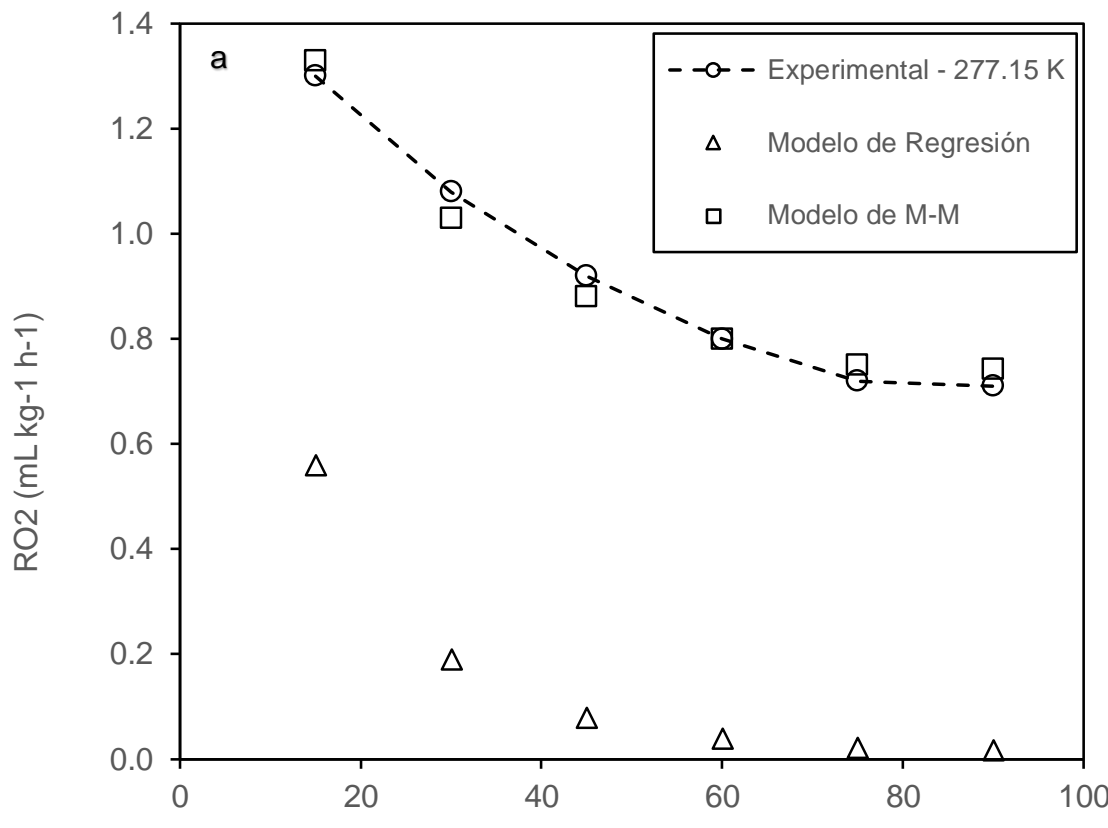

Tiempo (h)

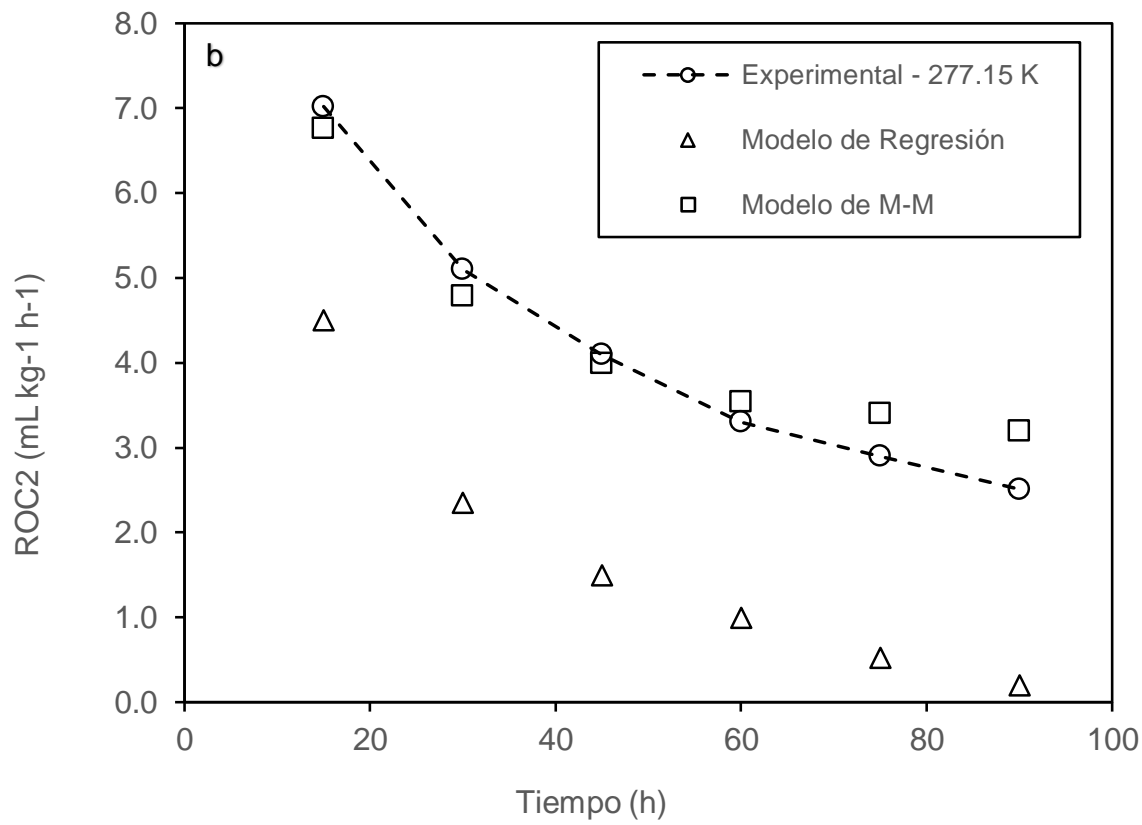

Fig. 2: Velocidad de respiración del mango cortado OD a 277.15 K. a) Consumo de $\mathrm{O}_{2}\left(\mathrm{R}_{\mathrm{O} 2}\right)$ b) Producción del $\mathrm{CO}_{2}\left(\mathrm{R}_{\mathrm{cO} 2}\right)$. - - velocidad experimental (Ec. 1 y 2 ); velocidad predicha con $\square \mathrm{M}-\mathrm{M} ; \boldsymbol{\Delta} \mathrm{M}-\mathrm{R}$.

Velocidades de respiración estimadas con el modelo de regresión

A partir de los coeficientes obtenidos con el M-R que describe el comportamiento de los gases (Tabla 2), se calculó las velocidades de respiración Ro2 y Rco2, reemplazando los resultados de las ecuaciones (7) y (8) en las ecuaciones (9) y (10), respectivamente. En las Figuras 2, 3 y 4, se observa que el M-R presentó mayor ajuste a $308.15 \mathrm{~K}$ para $R_{\mathrm{co} 2}\left(\mathrm{R}^{2}=0.93\right)$, ese mismo comportamiento fue observado por Zapata et al. (2018) y Agudelo et al. (2016), quienes ajustaron este modelo en mango Tommy atkins cortado fresco y entero, respectivamente.

Los parámetros de la ecuación de M-M estimados con las ecuaciones (13) y (14) para la respiración del mango $\mathrm{OD}$ a las tres temperaturas, al igual que los $\mathrm{R}^{2}$ de los diferentes ajustes del modelo, se presentan en la Tabla 4. Al contrastar los valores de Ro2 y Rco2 predichos por M-M con aquellos obtenidos experimentalmente, se puede apreciar un buen ajuste de los mismos para ambas velocidades de respiración, principalmente a 277.15 $\mathrm{K}\left(\mathrm{R}^{2}=0,93\right.$ y 0,96$)$ y $293.15 \mathrm{~K}\left(\mathrm{R}^{2}=0,96\right.$ y 0,99$)$. Los valores de $\mathrm{Km}$ decrecen con el aumento de la 
temperatura, viéndose reducidas las afinidades de las enzimas por los sustratos en la reacción de respiración (Preetinder et al., 2011), con ese aumento de temperatura los valores de Ki incrementan con lo que el efecto inhibidor del $\mathrm{CO}_{2}$ se ve reducido. Por el contrario, valores de $\mathrm{Ki}<1$ la velocidad de respiración se hace más sensible a cambios del $\mathrm{CO}_{2}$. En la Tabla 4 se observa como el parámetro velocidad máxima (Vm) aumenta con la temperatura, lo que indica que se incrementa el punto de saturación de las enzimas que catalizan las reacciones de respiración, por lo que se ven favorecidas dichas reacciones con la temperatura (Preetinder et al, 2011).

En la Tabla 5 se presenta la Ea y Rp- determinados con la ecuación de Arrhenius para ambos gases entre 277.15 y $308.15 \mathrm{~K}$. Estos parámetros fueron utilizados para evaluar el comportamiento de la respiración en función del tiempo y la temperatura empleando dicho modelo, el cual presentó un buen ajuste a los datos experimentales de $R_{02}$ y $R_{\mathrm{co} 2}\left(R^{2}=0.93\right)$, cómo se puede apreciar en las figuras 2,3 y 4 .
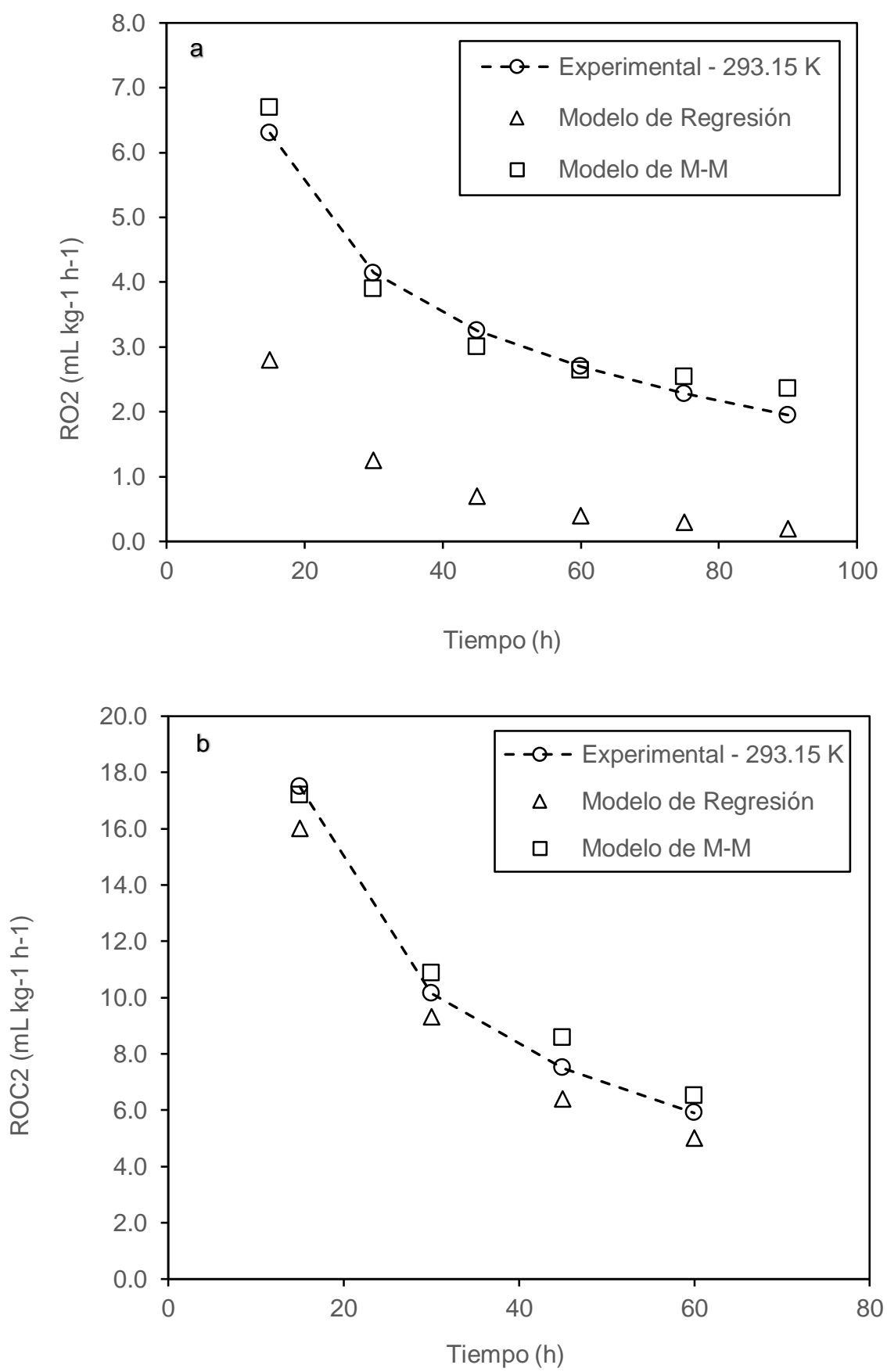

Fig. 3: Velocidad de respiración del mango cortado OD a 293.15 K. a) Consumo de O2 (RO2) b) Producción del CO2 (RCO2). - - velocidad experimental (Ec. 1 y 2); velocidad predicha con $\square \mathrm{M}-\mathrm{M} ; \mathbf{\Delta} \mathrm{M}-\mathrm{R}$. 

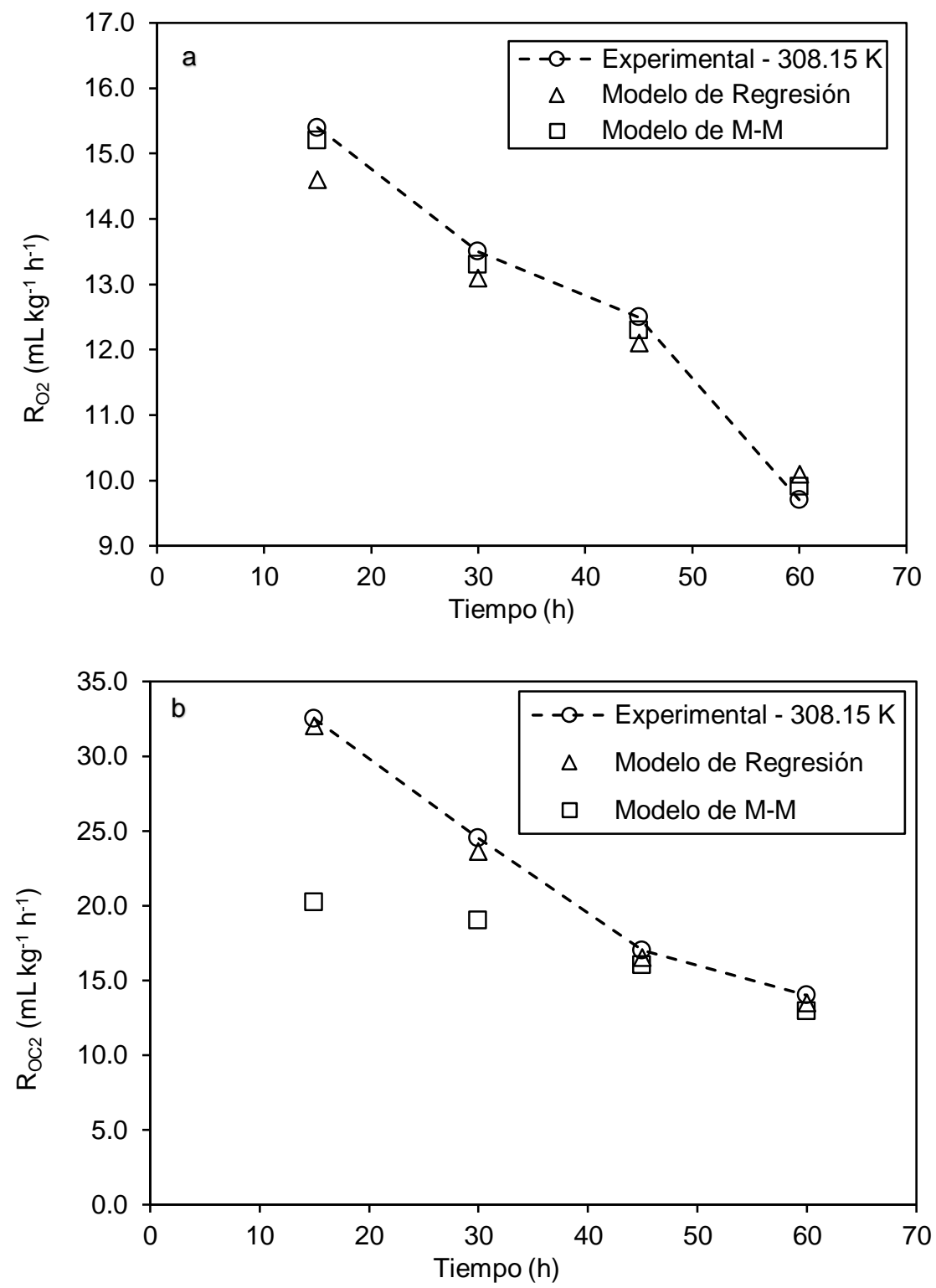

Fig. 4: Velocidad de respiración del mango cortado OD a 308.15 K. a) Consumo de O2 (RO2) b) Producción del CO2 (RCO2). - - velocidad experimental (Ec. 1 y 2); velocidad predicha con $\square \mathrm{M}-\mathrm{M} ; \mathbf{\Delta} \mathrm{M}-\mathrm{R}$.

Tabla 4: Parámetros del modelo de Michaelis-Menten con inhibición no competitiva en diferentes temperaturas, para mango osmodeshidratado

\begin{tabular}{|l|c|c|c|c|c|c|}
\hline \multirow{3}{*}{ Parámetros } & \multicolumn{6}{|c|}{ Temperatura $(\mathrm{K})$} \\
\cline { 2 - 7 } & \multicolumn{2}{|c|}{277.15} & \multicolumn{2}{|c|}{293.15} & \multicolumn{2}{c|}{308.15} \\
\cline { 2 - 7 } & $\mathrm{O}_{2}$ & $\mathrm{CO}_{2}$ & $\mathrm{O}_{2}$ & $\mathrm{CO}_{2}$ & $\mathrm{O}_{2}$ & $\mathrm{CO}_{2}$ \\
\hline $\mathrm{V}_{\mathrm{m}}\left(\mathrm{mL} \mathrm{kg}^{-1} \mathrm{~h}^{-1}\right)$ & 5.69 & 60.05 & 14.31 & 19.00 & 359.09 & 102.16 \\
\hline $\mathrm{K}_{\mathrm{m}}\left(\% \mathrm{O}_{2}\right)$ & 0.32 & 0.34 & 0.30 & 0.30 & 0.03 & -0.01 \\
\hline $\mathrm{K}_{\mathrm{i}}\left(\% \mathrm{CO}_{2}\right)$ & 1.19 & 0.47 & 0.10 & 1.17 & 174.12 & 917.68 \\
\hline $\mathrm{R}^{2}$ & 0.93 & 0.96 & 0.96 & 0.99 & 0.99 & 0.54 \\
\hline
\end{tabular}

Tabla 5: Parámetros de los modelos de Arrhenius para la predicción de la velocidad de respiración del mango OD

\begin{tabular}{|l|c|c|}
\hline Parámetros & $\mathrm{O}_{2}$ & $\mathrm{CO}_{2}$ \\
\hline$E a(\mathrm{~kJ} / \mathrm{g} \mathrm{mol})$ & 55.76 & 31.42 \\
\hline$R p$ & 3.53 & 10.05 \\
\hline $\mathrm{R}^{2}$ & 0.93 & 0.93 \\
\hline
\end{tabular}


El análisis de los valores de Ea, entendida como la cantidad de energía mínima requerida para iniciar una reacción, permite observar que la reacción de consumo de oxígeno requiere una cantidad de energía mayor, que la producción de $\mathrm{CO}_{2}$. Esto se debe a que Ro2 es más sensible a los cambios de temperatura que Rco2, posiblemente porque como ya se mencionó, el aumento de temperatura reduce la afinidad de las enzimas por los sustratos en la reacción de respiración y reduce el efecto inhibidor del $\mathrm{CO}_{2}$ (Preetinder et al., 2011).

\section{Efecto de la temperatura sobre la velocidad de respiración del mango}

El efecto de la temperatura sobre $\mathrm{R}_{\mathrm{O} 2}$ y $\mathrm{R}_{\mathrm{co} 2}$ es significativo porque acelera los procesos metabólicos de las células (Ghosh y Dash, 2018). En la Tabla 6 se presenta una comparación de los valores promedio de Ro2 y Rco2 experimentales y predichos por los modelos $M-R, M-M$ y Arrhenius, para el mango OD en las tres temperaturas evaluadas. Se observa que M-M y la ecuación de Arrhenius presentaron los valores más próximos a los datos experimentales, por lo tanto, fueron los de mejor ajuste a los mismos (Figuras 2, 3 y 4). Zapata et al. (2018) y Agudelo et al. (2016) también reportaron que M-R es el que presenta menor ajuste a los datos experimentales de mango entero y mango cortado fresco, respectivamente. A pesar de ese comportamiento en el ajuste de los datos, empleando dichos modelos, es importante resaltar que la ecuación de Arrhenius es única para cada gas y se puede usar en cualquier temperatura dentro del rango de trabajo, mientras que la ecuación del modelo M-M está parametrizada solo para trabajar a una temperatura específica y no para valores intermedios en el rango de temperaturas evaluadas en este estudio, por tanto, tiene sus limitaciones.

Tabla 6: Velocidades promedio de consumo de $\mathrm{O}_{2}\left(\mathrm{R}_{\mathrm{O} 2}\right)$ y producción de $\mathrm{CO}_{2}\left(\mathrm{R}_{\mathrm{CO} 2}\right)$ de los modelos a diferentes temperaturas en mango OD. Valores promedio \pm desviación estándar $(n=3)$

\begin{tabular}{|c|c|c|c|c|c|}
\hline Temperatura (K) & Gas & Experimental & $\mathrm{M}-\mathrm{R}$ & $\mathrm{M}-\mathrm{M}$ & Arrhenius \\
\hline \multirow{2}{*}{277.15} & $\mathrm{CO}_{2}$ & $4.09 \pm 1.51$ & $1.81 \pm 1.48$ & $4.33 \pm 1.42$ & $4.85 \pm \mathrm{na}$ \\
\cline { 2 - 6 } & $\mathrm{O}_{2}$ & $1.53 \pm 0.70$ & $0.16 \pm 0.11$ & $0.93 \pm 0.27$ & $0.97 \pm \mathrm{na}$ \\
\hline \multirow{2}{*}{293.15} & $\mathrm{CO}_{2}$ & $8.66 \pm 5.19$ & $1.75 \pm 1.15$ & $8.84 \pm 1.06$ & $10.20 \pm \mathrm{na}$ \\
\cline { 2 - 6 } & $\mathrm{O}_{2}$ & $3.41 \pm 1.62$ & $0.96 \pm 0.57$ & $2.97 \pm 0.39$ & $3.63 \pm \mathrm{na}$ \\
\hline \multirow{2}{*}{308.15} & $\mathrm{CO}_{2}$ & $18.36 \pm 0.23$ & $18.04 \pm 8.47$ & $18.74 \pm 0.10$ & $19.11 \pm \mathrm{na}$ \\
\cline { 2 - 6 } & $\mathrm{O}_{2}$ & $10.86 \pm 3.52$ & $1.84 \pm 1.26$ & $12.64 \pm 0.96$ & $11.05 \pm \mathrm{na}$ \\
\hline
\end{tabular}

La comparación del ajuste con el modelo de regresión (Tabla 2) y el modelo global (Tabla 7), en términos de la capacidad de predecir el comportamiento de los gases, parecen indicar un ajuste superior para el modelo de regresión, sin embargo, es útil solo para las condiciones de temperatura específica en las que fueron determinados los parámetros, por ser un modelo empírico, mientras que el modelo global por ser un modelo semi-empírico es más robusto y permite hacer predicción en todo el rango de temperaturas y tiempos del estudio, que son las convencionales para el almacenamiento del mango.

Tabla 7: Parámetros del modelo Global para la predicción de $\mathrm{R}_{\mathrm{O} 2}$ y $\mathrm{R}_{\mathrm{CO} 2}$ del mango OD

\begin{tabular}{|c|c|c|}
\hline Parámetros & $\mathrm{O}_{2}$ & $\mathrm{CO}_{2}$ \\
\hline Ea $(\mathrm{kJ} / \mathrm{g} \mathrm{mol})$ & 67.34 & 40.38 \\
\hline$R p$ & 2.66 & 8.12 \\
\hline $\mathrm{R}^{2}$ & 0.96 & 0.96 \\
\hline
\end{tabular}

En resumen, puede afirmarse que el modelo de M-M es útil para determinar el efecto de la concentración de gases sobre la velocidad de consumo de $\mathrm{O}_{2}$ y producción de $\mathrm{CO}_{2}$, el modelo de Arrhenius para evaluar la respiración en función de la temperatura y el modelo global para determinar la concentración de gases en función del tiempo y la temperatura simultáneamente.

\section{CONCLUSIONES}

Es posible reducir el contenido de agua en el mango en más del $61 \%$ e incrementar los sólidos solubles por encima del $24 \%$ en $4 \mathrm{~h}$ de tratamiento osmótico, bajo las condiciones del presente estudio. La concentración de los gases al interior del sistema cerrado va sufriendo modificaciones en función del tiempo y la temperatura que pueden ser predichas por el modelo semi-empírico global presentado en este estudio. La velocidad de respiración depende de la concentración de gases y de la temperatura, así mismo puede ser predicha en términos del consumo de $\mathrm{O}_{2}$ y producción de $\mathrm{CO}_{2}$ utilizando el modelo de $\mathrm{M}-\mathrm{M}$ y en función de la temperatura empleando el modelo de Arrhenius. 


\section{AGRADECIMIENTOS}

Los autores del presente trabajo agradecen a la Universidad de Antioquia por el apoyo financiero y a la Fundación Intal por el apoyo logístico, prestado para el desarrollo de las actividades.

\section{NOTACIÓN}

\section{Símbolos}

PA = pérdida de agua, $\%$

GS = ganancia de sólidos solubles, $\%$

$\mathrm{M}_{\mathrm{i}}=$ masa inicial, $\mathrm{g}$

$\mathrm{M}_{\mathrm{f}}=$ masa final, $\mathrm{g}$

$x_{w i}=$ fracción de agua inicial

$x_{\mathrm{wf}}=$ fracción de agua final

$\mathrm{x}_{\mathrm{si}}=$ fracción de sólidos solubles inicial

$\mathrm{x}_{\mathrm{sf}}=$ fracción de sólidos solubles final

$\mathrm{R}_{\mathrm{O} 2}$ y $\mathrm{R}_{\mathrm{CO} 2}=$ tasas de respiración $\mathrm{mL} \mathrm{O}_{2} \mathrm{~kg}^{-1} \mathrm{~h}^{-1}$ y mL $\mathrm{CO}_{2} \mathrm{~kg}^{-1} \mathrm{~h}^{-1}$ respectivamente

$\left[\mathrm{O}_{2}\right]_{\mathrm{t}}$ y $\left[\mathrm{CO}_{2}\right]_{\mathrm{t}}=$ fracciones másicas al tiempo $\mathrm{t}$ de $\mathrm{O}_{2}$ y $\mathrm{CO}_{2}$, respectivamente

$\left[\mathrm{O}_{2}\right]_{\mathrm{t}+1}$ y $\left[\mathrm{CO}_{2}\right]_{\mathrm{t}+1}=$ fracciones másicas de $\mathrm{O}_{2}$ y $\mathrm{CO}_{2}$, respectivamente, en la medición siguiente al tiempo $\mathrm{t}$.

$\mathrm{t}=$ tiempo, $\mathrm{h}$

$\Delta \mathrm{t}=$ es la diferencia de tiempo entre dos mediciones del respectivo gas (h)

$\mathrm{V}=$ volumen libre, $\mathrm{mL}$

$\mathrm{W}=$ masa de la fruta, $\mathrm{Kg}$

$a$ y $b=$ coeficientes

$\mathrm{V}_{\mathrm{mo}}=$ velocidad máxima de respiración para el consumo de $\mathrm{O}_{2}, \mathrm{~mL} \mathrm{~kg}^{-1} \mathrm{~h}^{-1}$

$\mathrm{V}_{\mathrm{mc}}=$ velocidad máxima de respiración para el consumo de $\mathrm{CO}_{2}, \mathrm{~mL} \mathrm{~kg}^{-1} \mathrm{~h}^{-1}$

$\mathrm{K}_{\mathrm{mo}}=$ constante de Michaelis-Menten para el consumo de $\mathrm{O}_{2}, \%$

$\mathrm{K}_{\mathrm{mc}}=$ constante de Michaelis-Menten para la producción de $\mathrm{CO}_{2}$, \%

$\mathrm{K}_{\mathrm{io}}=$ constante de inhibición para el consumo de $\mathrm{O}_{2}, \%$

$\mathrm{K}_{\mathrm{ic}}=$ constante de inhibición para la producción de $\mathrm{CO}_{2}, \%$

$\mathrm{R}_{\text {gas }}=$ velocidad de consumo de $\mathrm{O}_{2}$ y producción de $\mathrm{CO}_{2}, \mathrm{~mL} \mathrm{~kg}^{-1} \mathrm{~h}^{-1}$

$\mathrm{R}_{\mathrm{p}}=$ factor pre-exponencial de la respiración

$\mathrm{E}_{\mathrm{a}}=$ energía de activación, $\mathrm{kJ} \mathrm{g}^{-1} \mathrm{~mol}^{-1}$

$\mathrm{R}=$ constante universal de los gases, $8.314 \mathrm{Jg}^{-1} \mathrm{~mol}^{-1} \mathrm{~K}^{-1}$

$\mathrm{T}_{\text {abs }}=$ temperatura absoluta, $\mathrm{K}$

Abreviaciones

$\mathrm{DO}=$ deshidratación osmotica

$\mathrm{OD}=$ Osmodeshidratado

NTC $=$ Norma Técnica Colombiana

$\mathrm{M}-\mathrm{R}=$ modelo de regresión

$\mathrm{M}-\mathrm{M}=$ Michaelis-Menten

\section{REFERENCIAS}

Agudelo, C., Restrepo, C., y Zapata, J. E., Respiration Kinetic of Mango (mangifera indica I.) as Function of Storage Temperatura, http://doi.org/10.15446/rfna.v69n2.59143, Revista Facultad Nacional de Agronomía Medellín, 69(2), 79857995 (2016).

AOAC, 934.06 y 942.15 Official Methods of Analysis, 14th edition, Gaithersburg, Estados Unidos (1984).

Arias, L., Perea, Y., y Zapata, J. E., Cinética de la Transferencia de Masa en la Deshidratación Osmótica de Mango (Mangifera indica L.) var. Tommy Atkins en Función de la Temperatura. http://doi.org/10.4067/S071807642017000300006, Inf. Tecnol, 28(3), 47-58 (2017).

Artés-Hernández, F., y Conesa, A., Minimally Fresh Processed Pepper Under Different Kind of Cuts, http://doi.org/10.17660/ActaHortic.2010.857.1, Acta Hortic, 857(1), 25-30 (2010).

Bhande, S. D., Ravindra, M. R., y Goswami, T. K., Respiration rate of Banana Fruit Under Aerobic Conditions at Different Storage Temperatures, http://doi.org/10.1016/j.jfoodeng.2007.11.019, J Food Eng, 87(1), 116-123 (2008).

Blanco-Díaz, M. T., Pérez-Vicente, A., y Font, R., Quality of Fresh Cut Zucchini as Affected by Cultivar, Maturity at Processing and Packaging, http://doi.org/10.1002/pts.2214, Packag Technol Sci, 29(7), 365-382 (2016).

Buitrago-Dueñas, E. M., Dussán-Sarria, S., y otros dos autores, Efecto del tipo de corte y tipo de envase en la conservación de piña [Ananas comosus (L.) Merr.] 'Oro Miel' mínimamente procesada, https://doi.org/10.17584/rcch.2018v12i2.7485, Revista Colombiana de Ciencias Hortícolas, 12(2), 308-318 (2018).

Carvajal, L. M., Ospina de Barreneche, N., y Zapata, J. E., Aplicación de Métodos Combinados Para la Conservación de Papaya Hawaiana (Carica papaya) Cortada en Láminas, Alimentación, Equipos y Tecnología, ISSN 0212-1689, 190(23), 113-120 (2004). 
Castellanos, D., "Evaluación del Almacenamiento de Bananito (Musa acuminata AA) con Atmósferas Modificadas" Tesis de Magister en Ciencia y Tecnología de Alimentos, Facultad de Ciencias Agrarias, Universidad Nacional - Colombia (2012).

Chiumarelli, M., Ferrari., C. C., y otros cuatro autores, Fresh Cut "Tommy Atkins" Mango Pre-Treated With Citric Acid and Coated With Cassava (Manihot esculenta Crantz) Starch or Sodium Alginate. http://doi.org/10.1016/j.ifset.2011.02.006, Innovative Food Sci Emerg Technol, 12(3), 381-387 (2011).

Devanesan, J. N., Karuppiah, A., y Abirami, C. V., Effect of Storage Temperatures, $\mathrm{O}_{2}$ Concentrations and Variety on Respiration of Mangoes, http://doi.org/10.2478/v10146-011-0013-8, Journal of Agrobiology, 28(2), 119-128 (2011).

Ghosh, T., y Dash, K. K., Respiration Rate Model and Modified Atmosphere Packaging of Bhimkol Banana, http://doi.org/10.1016/j.eaef.2018.04.004, Eng Agric Environ Food, 11(4), 186-195 (2018).

ICONTEC, Norma Técnica Colombiana 5210, Frutas frescas. Mango. Variedades mejoradas. Especificaciones, 1-33, Bogotá, Colombia (2003).

Iqbal, T., Rodrigues, F., y otros dos autores, Mathematical Modelling of the Influence of Temperature and Gas Composition on the Respiration Rate of Shredded Carrots, https://doi.org/10.1016/j.jfoodeng.2008.09.012, J Food Eng, 91(2), 325-332 (2009).

Mangaraj, S., y Goswami, T. K., Measurement and Modeling of Respiration Rate of Guava (CV. Baruipur) for Modified Atmosphere Packaging, http://doi.org/10.1080/10942910903312403, Int J Food Prop, 14(3), 609-628 (2011).

Nicolaï, B., Hertog, M., y otros dos autores, Gas Exchange Modeling. In Modified and Controlled Atmospheres for the Storage, Transportation, and Packaging of Horticultural Commodities by Yahia. E. pp 93-108 CRC Press, New York, USA, (2009).

Ntsoane, M. L., Zude-Sasse, M., y otros dos autores, Quality assesment and postharvest technology of mango: A review of its current status and future perspectives, https://doi.org/10.1016/j.scienta.2019.01.033, Sci Hort, 249 (abril), 77-85 (2019).

Pan, Y., Zhu J., y Li S, Effects of pure oxygen and reduced oxygen modified atmosphere packaging on the quality and microbial characteristics of freshcut pineapple, http://doi.org/10.1051/ fruits/2015003, Fruits, 70(2), 101-108 (2015).

Preetinder, K., Deepak, R. R., y Shashi, P. Nonlinear Estimation of Respiratory Dynamics of Fresh-Cut Spinach (spinacia oleracea) Based on Enzyme Kinetics, http://doi.org/10.1111/j.1745-4530.2009.00508, J Food Process Eng, 34(1), 21372155 (2011).

Qin, G. Z., Wang, Q., y otros tres autores, Proteomic Analysis of Changes in Mitochondrial Protein Expression During Fruit Senescence. http://doi.org/10.1002/pmic.200900133, Proteomics, 9(1), 4241-4253 (2009).

Ravindra, M. R., y Goswami, T. K., Modelling the Respiration Rate of Green Mature Mango Under Aerobic Conditions, http://doi.org/10.1016/j.biosystemseng.2007.10.005, Biosyst Eng, 99(2), 239-248 (2008).

Ríos, M., Márquez, C. J., y Ciro, H., Deshidratación Osmótica de Frutos de Papaya (Carica papaya L.) Hawaiiana en Cuatro Agentes Edulcorantes, Revista Facultad Nacional de Agronomía Medellín, ISSN 0304-2847, 58(2), $2989-3002$ (2005).

Sanjinez-Argandoña, E. J., Yahagi, L. Y., y otros dos autores, Mango Dehydration: Influence of Osmotic Pre-Treatment and Addition of Calcium Chloride, http://doi.org/10.1590/0100-29452018419, Revista Brasileira de Fruticultura, 40(4), 410419 (2018).

Selcuk, N., y Erkan, M., The Effects of Modified and Palliflex Controlled Atmosphere Storage on Postharvest Quality and Composition of 'Istanbul' Medlar Fruit, http://doi.org/10.1016/j.postharvbio.2014.07.004, Postharvest Biol Technol, 99(1), 9-19 (2015)

Zapata, J. E., Agudelo, C., y Restrepo, C., Modelamiento de la Respiración del Mango (Mangifera indica L.) Usando el Método de Sistema Cerrado a Diferentes Temperaturas, http://doi.org/10.1590/0100-29452018126, Revista Brasileira de Fruticultura, 40(3), 112 - 126 (2018).

Zuluaga, J. D., Cortes-Rodríguez, M., y Rodríguez-Sandoval, E., Evaluación de las Características Físicas de Mango Deshidratado Aplicando Secado por Aire Caliente y Deshidratación Osmótica, Revista de la Facultad de Ingeniería Universidad Central de Venezuela, ISSN 0798-4065, 25(4), 127-135 (2010). 
